\title{
Das kritische Gutachten des Wissenschaftlichen Beirats zur humanistischen Psychotherapie: Anlass zur Reflexion des Begriffs "Psychotherapieverfahren"
}

\author{
Winfried Rief \\ AG Klinische Psychologie und Psychotherapie, Fachbereich Psychologie, Philipps-Universität Marburg, Marburg, Deutschland
}

Der Wissenschaftliche Beirat Psychotherapie (WBP), ein mit hochrangigen Vertretern der Psychotherapieforschung besetztes offizielles Gremium, hat seine Bewertung des Antrags auf Anerkennung der «humanistischen Therapien» als wissenschaftlich fundiertes Verfahren abgegeben. In diesem Antrag wurden 10 unterschiedliche therapeutische Ansätze zusammengefasst, die von der Gesprächspsychotherapie über die Gestalttherapie bis hin zur Bioenergetik reichten. Zum einen wurde im Gutachten des WBP festgestellt, dass es sich bei der humanistischen Psychotherapie nicht um ein Psychotherapieverfahren im engeren Sinne handelt, da die konzeptionelle Basis sowohl hinsichtlich der Ätiologiemodelle für psychische Störungen als auch der Behandlungskonzepte zu heterogen ist; zum anderen wurde konstatiert, dass keiner der genannten Ansätze die Kriterien für eine wissenschaftliche Anerkennung als Verfahren verdient. Selbst die Gesprächspsychotherapie, bei der die Anerkennung in einer früheren Stellungnahme knapp (mit einem Minderheitenvotum) befürwortet wurde, erfuhr in der Terminologie des WBP ein «Downgrading» zu einer Therapiemethode. Nur für 3 der vom WBP gelisteten 18 Indikationsbereiche wurden ausreichende Wirksamkeitsnachweise gesehen, die sich ausschließlich auf das Erwachsenenalter beziehen.

Die Stellungnahme hat viel Kritik vonseiten der Antragssteller und von Vertretern der entsprechenden psychotherapeutischen Ansätze ausgelöst. Teilweise wird dem Beirat und seinen Mitgliedern Parteilichkeit unterstellt, teilweise werden deren Arbeit und Wissenschaftsbegriff grundsätzlich infrage gestellt. Neben diesen archaisch anmutenden Reaktionen kann die Stellungnahme des WBP jedoch auch dazu anregen, zu reflektieren, ob nicht sowohl der gängige Begriff «Psychotherapieverfahren» als auch viele der damit verbundenen Vorstellungen von abgegrenzten, auf eigenen Theorien fundierenden Verfahren hinterfragt werden müssen. Zwar sind die Vertreter der Verhaltenstherapie, die bei allen wesentlichen Indikationsgebieten ausreichende wissenschaftliche Nachweise erbringen konnte, sozusagen auf der sicheren Seite, trotzdem wird zunehmend deutlich, dass manche der hier ange- sprochenen Probleme zu zunehmenden Konflikten für die gesamte Profession führen werden und neue Herangehensweisen notwendig machen. Wer oder was ist also «Schuld» an der konflikthaften Zuspitzung bei der Frage, welche Psychotherapieverfahren als wissenschaftlich anerkannt zu werten sind?

Der Hintergrund der wissenschaftlichen Prüfung eines Verfahrens sind die Versorgung und der Schutz von Patienten. Es geht um die Frage, ob Psychotherapeuten nach der Ausbildung in einem bestimmten Verfahren die Kompetenzen besitzen, um das Gesamtspektrum der für eine Psychotherapie infrage kommenden Patienten verantwortungsvoll und auf wissenschaftlich fundierter Grundlage zu behandeln. Aber ist die Zielsetzung, ein Psychotherapieverfahren pauschal und gegebenenfalls für alle Zeiten und für alle Indikationsbereiche zu legitimieren, überhaupt sinnvoll? Diese Intention wäre legitim, wenn tatsächlich davon auszugehen wäre, dass sich durch den Nachweis der wissenschaftlichen Fundierung eines Verfahrens in einigen wenigen Anwendungsbereichen automatisch auch auf eine Wirksamkeit dieses Verfahren in den restlichen Indikationsbereichen der entsprechenden Altersspanne schließen lässt, und dass dieses dann dort ebenfalls zu den Spitzenempfehlungen zählt. Eine solche Annahme ist wissenschaftlich und praktisch klar zurückzuweisen. So haben wir bei der Behandlung von Depressionen durchaus verschiedene Behandlungsansätze, die theoretisch gut fundiert sind und deren Wirksamkeit empirisch belegt ist, die jedoch nicht automatisch auch bei schwer ausgeprägten chronifizierten Zwangserkrankungen oder während akuter psychotischer Phasen als hilfreich zu bewerten sind. Somit ist die oben erwähnte Grundidee, einem Verfahren für jetzt und möglichst für alle Zeiten und alle Indikationen eine wissenschaftliche Anerkennung auszusprechen, falsch. Demgegenüber müsste die wissenschaftliche Anerkennung ein problemspezifischer und zeitlich befristeter Beschluss sein: Wenn bei einem bestimmten Indikationsgebiet eine wissenschaftliche Anerkennung entsprechend den aktuell gültigen Qualitätskriterien ausgesprochen wird, muss diese unter Umständen einige Jahre später wieder revidiert wer-

\section{KARGER}

(c) 2018 S. Karger GmbH, Freiburg

Fax +497614520714
Prof. Dr. Winfried Rief

AG Klinische Psychologie und Psychotherapie, Fachbereich Psychologie

Philipps-Universität Marburg

Gutenbergstraße 18, 35032 Marburg, Deutschland

rief@staff.uni-marburg.de 
Kasten 1. Zum

Nachdenken
Die Hauptfrage kann nicht sein, ob ein Verfahren bei 3 oder 4 von 12 klinisch relevanten Indikationsgebieten des Altersbereichs wissenschaftlich fundierte Ansätze anbietet - sondern, was mit den anderen Indikationsgebieten ist. Wie werden in der Ausbildung zu einem Schwerpunktverfahren Kompetenzen dafür erworben, auch in Indikationsgebieten wissenschaftlich fundierte Interventionen durchzuführen, für die das Schwerpunktverfahren nicht zu den wissenschaftlich fundierten Behandlungsempfehlungen zählt? den, weil es bessere Behandlungsansätze gibt, weil die Wissenschaft strengere Kriterien für die Anerkennung von Studien fordert oder weil das klinische Problem differenzierter bewertet werden muss.

Für die Politik und das Rechtssystem im Gesundheitswesen, insbesondere in Bezug auf das Berufs- und Sozialrecht, hat das Festhalten an traditionellen Verfahrensbegriffen gewisse Vorteile: Es schafft eine vermeintliche Klarheit, die sich auch juristisch regeln lässt. Insofern ist bis hin zum Bundesministerium für Gesundheit auch die Politik dafür verantwortlich, dass in Rechtsprechungen am traditionellen Verständnis von Psychotherapieverfahren festgehalten wird und - schlimmer noch - dass manche der Verfahren dann per Gesetz zu Richtlinienverfahren erklärt und damit offensichtlich bevorteilt werden. Ein wichtiger Schritt bestand darin, dass auch die Richtlinienverfahren sich teilweise der Prüfung des WBP gestellt haben, um hier Vergleichbarkeit herzustellen. Allerdings geschah dies nicht in allen relevanten Gebieten: Für psychodynamische oder psychoanalytische Verfahren zur Behandlung von Kindern und Jugendlichen hat der WBP bislang keine wissenschaftliche Anerkennung erteilt - hier weisen Vertreter anderer Verfahren zu Recht auf Ungerechtigkeiten hin.

Natürlich wird nun auch auf die Psychotherapieforscher gezeigt: Machen sie die falschen Studien, berücksichtigen sie die traditionellen Verfahren zu wenig? Die Dynamik der (Psychotherapie-)Forschung folgt in der Tat anderen Regeln, als sich mancher Psychotherapieverband wünschen mag. Gerade die Gesprächstherapie war z.B. in den 1970er Jahren ein wissenschaftlich interessantes Betätigungsfeld, und zahlreiche Professoren haben sich ihr gewidmet. Diese werden sich fragen müssen, ob sie genug für die Forschung und den wissenschaftlichen Nachwuchs in dem Bereich unternommen haben. Jedenfalls ist der Zug weitergefahren, und heute wird man Psychotherapieforscher leichter motivieren können, zu einem neuen Behandlungsansatz zu forschen, als zu einem traditionellen, das wissenschaftlich weniger ergiebig erscheint. Und ob bzw. wie stark durch externe Steuerung in die Freiheit der Forschung eingegriffen werden soll, will gut überlegt sein. Eine primäre Auftragsforschung mit offensichtlichen Interessenvorgaben kann hier kaum die Lösung sein.

Durch den starken Fokus auf eine generalisierte wissenschaftliche Anerkennung ergibt sich eine zum Teil drastische Diskrepanz zwischen den Anerkennungen von Psychotherapieverfahren und den in wissenschaftlich fundierten Leitlinien ausgesprochenen Behandlungsempfehlungen. Letztere geben eher den aktuellen wissenschaftlichen Stand wieder und gehen mit der Verpflichtung einher, in regelmäßigen Abständen aktualisiert zu werden. $\mathrm{Zu}$ allen größeren Indikationsgebieten der Psychotherapie weisen wissenschaftlich fundierte Behandlungsleitlinien in aller Regel mehrere
Verfahren, Methoden oder Techniken als «State of the Art» aus. Im Gegensatz zu allen anderen Teilgebieten der Medizin wird in der Psychotherapie aber nicht dahingehend ausgebildet, dass approbierte Psychotherapeuten die Kompetenz haben, wissenschaftlich fundierte State-of-the-Art-Interventionen bei allen wesentlichen klinischen Problemkonstellationen durchzuführen, sondern sie entscheiden sich in der Aus- oder Weiterbildung pauschal für ein Verfahren, das dann bei allen Indikationsgebieten angewandt werden soll, selbst wenn es per wissenschaftlichen Leitlinien nicht einmal zu den engeren Empfehlungen im Sinne von klinischen Konsenspunkten gehört.

Vor diesem Hintergrund ist auch beeindruckend, wie die aktuelle Diskussion um das Gutachten zur humanistischen Psychotherapie darum kreist, ob die Gesprächstherapie nun in 3 der 12 für den Erwachsenenbereich relevanten Indikationsgebieten als wissenschaftlich fundiert gewertet werden sollte - oder in 4 (siehe Kasten 1). Die Hauptfrage muss hier jedoch lauten: Soll man dieses (und potenzielle andere) Verfahren als eigenständiges Weiterbildungsverfahren zulassen, obwohl es in mindestens 8, vielleicht auch in 9 Indikationsgebieten keine wissenschaftlichen Wirkungsnachweise aufbringen konnte, und obwohl für diese 8-9 Indikationsbereiche klare, wissenschaftlich fundierte Empfehlungen für andere Interventionen vorliegen? Diese Frage stellt sich selbstverständlich genauso für andere, vom WBP positiv beschiedene Verfahren. Wäre hier nicht wie überall in der Medizin zu fordern, dass Weiterbildungen für alle klinisch relevanten Indikationsbereiche jeweils wissenschaftlich anerkannte, in Leitlinien empfohlene Behandlungen vermitteln sollen, obwohl hierzu gegebenenfalls Anleihen aus anderen Verfahren integriert werden müssten? Auch wenn nun viele Verfahrensvertreter aufschreien mögen: Für manche Psychotherapieverfahren wäre dies unter Umständen der einzige Weg, um zu überleben, anstatt die in vielen Fällen übergroße Hürde einer globalen wissenschaftlichen Anerkennung bei steigenden wissenschaftlichen Kriterien für Therapiestudien anzustreben.

Wenn es um die Verantwortung für den negativen Ausgang des Begutachtungsverfahrens durch den WBP zur humanistischen Psychotherapie geht, sind jedoch auch die wissenschaftlichen Berater der Antragssteller zu erwähnen. Wer die Psychotherapieforschung der letzten 20-30 Jahre ernsthaft verfolgt hat, konnte nicht davon ausgehen, dass sich die Situation für viele der unter dem Dach humanistischer Verfahren versammelten Behandlungsansätze substanziell gebessert hätte. Hierbei ist zu berücksichtigen, dass die Qualitätskriterien für die Anerkennung von Studien einem natürlichen Prozess und den wissenschaftlichen Erkenntnissen folgend kontinuierlich ansteigen (müssen); daraus ergibt sich als logische Konsequenz, dass viele ältere Studien nach und nach aus dem 
Qualitätsfenster herausfallen und nicht mehr gewertet werden können. Darauf hätten die wissenschaftlichen Berater hinweisen müssen. Und selbstverständlich haben auch die Antragssteller selbst eine gewisse Mitverantwortung für den negativen Ausgang: Der Versuch, die sowieso schon kritisch aufgestellte Gesprächspsychotherapie als Tragesel für zahlreiche weitere, wissenschaftlich fast gar nicht nach modernen Kriterien evaluierten Therapieansätze zu verwenden, führte dazu, dass der schwache Tragesel in die Knie ging.

Die Frage der konzeptionellen Kohärenz eines Verfahrens und seiner Abgrenzung zu anderen Verfahren stellt jedoch nicht nur beim Antrag für die humanistische Psychotherapie einen schwierigen Punkt dar, sondern wird zunehmend für alle Psychotherapieverfahren relevant. Die traditionelle Definition der Verhaltenstherapie als Interventionen, die auf der Lernpsychologie basieren und wissenschaftlich fundiert sind, ist genauso überaltert wie die Definition psychodynamischer Verfahren als Methoden, die primär mit dem Unbewussten arbeiten oder auf der ursprünglichen Theorie der Psychoanalyse aufbauen. Manche sogenannten «DritteWelle»-Verfahren werden der Verhaltenstherapie zugeordnet, obwohl sie konzeptionell genauso gut als psychodynamische Verfahren betrachtet werden können oder letztendlich eine eigene Gruppe bilden müssten (z.B. Schematherapie, Cognitive Behavioral Analysis System of Psychotherapy (CBASP)). Manche Behandlungsansätze haben ganz eigene theoretische und historische Wurzeln (z.B. achtsamkeitsbasierte Verfahren), die mit denen der gängigen anderen Verfahren wenig gemeinsam haben. Während verfahrensspezifische Vorgehensweisen in den 1960er Jahren teilweise noch klar zu unterscheiden waren, sind mindestens die beiden Großfamilien psychotherapeutischer Richtungen (psychodynamische Verfahren und kognitive Verhaltenstherapie (KVT)) zwischenzeitlich durch eine hohe interne Heterogenität der Interventionen gekennzeichnet, die an den Rändern stark überlappen. Außerdem kommen immer mehr verfahrensübergreifende Vorgehensweisen hinzu, die eine klare Abgrenzung zu einer Schimäre machen.

Ein interessanter Aspekt am Rande: Für Verfahren wie die Acceptance and Commitment Therapy oder achtsamkeitsbasierte Interventionen gibt es indessen jeweils zwischen 70 und 140 randomisierte klinische Studien, die mindestens die Behandlung epidemiologisch wesentlicher Störungen wie Depression, Angsterkrankungen, Traumatisierung und somatische Beschwerden abdecken [Gotink et al., 2015; Ost, 2014]. Diese beiden Verfahren haben somit eine Evidenzlage, die stärker ist als bei allen einzelnen nichtverhaltenstherapeutischen Verfahren, und müssten bei einer Prüfung durch den WBP vermutlich anerkannt werden. Durch das in Deutschland an traditionellen Verfahren orientierte System verzögert sich deren Einführung und Anerkennung jedoch hierzulande deutlich im Vergleich z.B. zu den USA, den Niederlanden, Großbritannien oder den skandinavischen Ländern. Hier stellt sich die Frage, ob unser System in Deutschland flexibel genug ist, um zeitnah wissenschaftlich fundierte therapeutische Innovationen $\mathrm{zu}$ integrieren, oder ob systemkonservativ veraltete Verfahrensbegriffe gepflegt werden.
Wozu sollte uns dieser kritische Bescheid zur humanistischen Psychotherapie nun also motivieren? Zum einen müssen wir alle das Konzept von (in sich abgeschlossenen und konzeptionell stimmigen) Therapieverfahren hinterfragen. Diese Abgrenzung einzelner Verfahren mag vor 30 oder 50 Jahren noch einfach gewesen sein, heute ist sie aber letztendlich unmöglich. Zum anderen muss die strenge Orientierung der Therapieaus- und -weiterbildung an einem einzelnen Verfahren hinterfragt werden: Das Ziel einer Weiterbildung in der Medizin besteht grundsätzlich darin, die Kompetenz zur Durchführung von Behandlungen aller wesentlichen Erkrankungen der Spezialisierung entsprechend den wissenschaftlich fundierten Leitlinien zu erwerben. Will man die «kleineren» Verfahren retten, müssen diese für die Teilgebiete in der Weiterbildung zugelassen werden, für die sie eine ausreichende Evidenz aufweisen; für die weiteren Teilgebiete müssen dann Anleihen bei anderen wissenschaftlich fundierten Behandlungsansätzen zugelassen werden, die nicht dem eigenen Verfahren zuzuordnen sind, aber in wissenschaftlichen Behandlungsleitlinien empfohlen werden. Den Fachkundenachweis als approbierter Psychotherapeut kann nur jemand erhalten, der die Kompetenzen zur Behandlung aller wesentlichen Problemkonstellationen, bei denen eine Psychotherapie indiziert ist, nach wissenschaftlich bestem Kenntnisstand besitzt. So stehen für die Behandlung von Depressionen zahlreiche wissenschaftlich fundierte Behandlungsmöglichkeiten zur Auswahl: KVT, interpersonelle Psychotherapie, gegebenenfalls psychodynamische und gesprächstherapeutische Ansätze, bei rezidivierender Depression vielleicht auch achtsamkeitsbasierte Ansätze oder CBASP bei chronifizierten Depressionen. Bei einzelnen anderen Krankheitsbildern, wie z.B. Psychosen vom schizophrenen Formenkreis, wird die Auswahl an wissenschaftlich fundierten Interventionen deutlich geringer ausfallen (vermutlich KVT und familientherapeutische Ansätze), und bei einer Aus- und Weiterbildung nach wissenschaftlich fundierten Ansätzen müsste den angehenden Psychotherapeuten dann zugemutet werden, die Kompetenz zur Durchführung einer solchen Behandlung bei dieser Indikation im Rahmen der Gesamtqualifikation zu erwerben, wiewohl sonst der Schwerpunkt auf anderen Verfahren läge. Auch wenn der Aufschrei an verschiedenen Stellen schon so gut wie hörbar ist: Wäre dies nicht eine professionelle und kompetente, der Patientenversorgung dienende Psychotherapieaus- oder -weiterbildung?

Es kann keine Lösung sein, die wissenschaftlichen Qualitätskriterien von Studien aufzuweichen oder persönlichen Interessen anzupassen - dies verbietet schlichtweg der Patientenschutz, der auch entsprechend den Festlegungen im Sozialgesetzbuch fordert, dass Behandlungen nach neuesten wissenschaftlichen Erkenntnissen durchgeführt werden. Die wissenschaftlichen Qualitätskriterien für diese Anerkennung sind veränderlich, und die Ansprüche werden naturgemäß höher, was der WBP bei seiner Bewertung berücksichtigen muss. Deshalb kann kein Verfahren, auch nicht die Richtlinienverfahren, für immer eine wissenschaftliche Anerkennung für alle Indikationsgebiete erhalten: Verfahren müssen kontinuierlich evaluiert und immer wieder insgesamt und für einzelne Indikationsgebiete neu bewertet werden. Hier benötigen wir einen dynamischeren und schnelleren Prozess als bisher. 
Den WBP für seine aktuelle Entscheidung zum Buhmann zu machen, dient niemandem. Das Gremium hat seine Aufgabe transparent durchgeführt, es ist mit Experten der Psychotherapieforschung besetzt und hat deshalb wie vom Gesetzgeber vorgesehen gehandelt. Insofern hat der WBP seine Aufgabe erfüllt. Das Urteil ist für alle, die sich in der Psychotherapieforschung der letzten 20 Jahre auskennen, keine Überraschung. Aber der WBP wird gefordert sein, seinen Kriterienkatalog dynamischer an aktuelle Entwicklungen der Psychotherapieforschung anzupassen, was jedoch der Gegenstand einer anderen Diskussion sein soll. Und wir alle sind gefordert, uns von den traditionellen, stark abgrenzenden Vorstellungen von Therapieverfahren zu lösen und neue Ord- nungskriterien zu entwickeln. Dabei darf das Hauptziel nicht aus den Augen verloren werden: Patientenschutz und Psychotherapie nach bestem, möglichst aktuellem wissenschaftlich fundiertem Wissen.

\section{Disclosure Statement}

Der Autor erklärt, keine finanziellen Interessenkonflikte zu haben, die die hier geäußerten Überlegungen beeinflusst haben könnten. Aus Gründen der Transparenz weist er darauf hin, dass er ebenfalls einen Großteil seiner Psychotherapieausbildung (jedoch nicht die gesamte) in einem Verfahren absolvierte und aktuell ein Psychotherapie-Ausbildungsinstitut leitet.

\section{Literatur}

Gotink RA, Chu P, Busschbach JJ, Benson H, Fricchione GL, Hunink MG: Standardised mindfulness-based in terventions in healthcare: an overview of systematic reviews and meta-analyses of RCTs. PLoS One 2015

Ost, LG: The efficacy of Acceptance and Commitment Therapy: an updated systematic review and meta-analysis. Behav Res Ther 2014;61:105-121.

10:e124344. 